\title{
Author Index Vol. 37, 1975
}

Adams, J. E. 279 Aiba, H. 259 Akagi, K. 120 Akil.H. 119 Amano, K. 285 Andy, O. J.

107, 215 Asakura, T. 78

Balasubramaniam, V. 195,

271 Bechtereva, N. P. 136 Beek, H. 166 Bertrand, C. 113 Black, P. 187 Bondartchuk, A.

N. 136 Bouchard, G. 232 Bronisch, F. 24

Crandall, P. 239

Davidson, A. 63 Dieckmann, G. 177 Doi, T. 156 Driollet, R. 9

Fairman, D. 172 Falconer, M. A. 243 Flanigin, H. 241 Fujita, Y. 265 Fukamachi, A. 258 Fukushima, T. 99

GajdoSova, D. 244 Gildenberg, Ph. 317 Goettsching, G. 207

Handa, H. 265 Hashimoto, I. 285 Hasse, G. R. 256 Hassler, R. 24,177, 329 
Heimburger, R. F. 16, 202,

270 Hirota, T. 156 fiënÏT. 99

Hosobuchi, Y. 279 Hughes, J. R. 215

Iiuzuka, J. 141 Ikeda, T. 302 Ishijima, B. 99 Ito, M. 265 Ito, Z. 41 Iwata, Y. 302

Jinnai, D. 302 Jurko, M. F. 215

Kalyanaraman, S. 189 Kamikawa, K. 120, 302 Kanaka, T. S. 195,271 Kandel,E.I. 128 Kawamura, H. 78 Kim, Y. K. 207, 232 Kimura, T. 302 Kitamura, K. 78 Kobayashi, K. 302 Kokavek, M. 244 Kooi, K.A. 165 Koshino, K. 223, 302 Krayenbühl, H. 357 Kudo, T. 97 Kullberg, G. 167 Kuramoto, S. 259 Kuroda, R. 120 Kwak, R. 249, 308

Lavallol, M. A. 172 Ldzér, L. 49 Lombard, S. A. 256

Martinez, S.N. 113 
Martin-Rodriguez, J. G. 56 Martins, L. F. 80 Matsumoto, K. 166 Matsumura, H. 223 Meliutcheva, L. A. 136 Meyer, J. S. 296 Mihara, T. 78 Miki, M. 223 Mimura, Y. 166

Miyamoto, T. 166 Miyazaki, H. 291 Mogami, H. 120, 302 Molina Negro, P. 113 Mori, K. 265 Moriyama, T. 291 Moriyasu, N. 10,280 Mukawa, J. 302 Mundinger, F. 24, 329 Murai, H. 120 Mutsuga, N. 156

Nadvornik. P. 193, 244 Nagao, I. 302 Nakamura, R. 258 Nakano, M. 223 Nakashima, H. 291 Narabayashi, H. 258, 364 Nashold, B. S. 163,241,374 Nittner, K. 133

Orlando, J. 9 Obrador, S. 56, 378 Ohye, Ch. 258 Okudaira, Y. 249, 308 Otabe, K. 308

Perez-Calvo, J. M. 113 Poblete, M. 150

Ramamurthi, B. 63,384 
Author Index

Richardson, D. E. 119 Riechert, T. 24, 329, 399 Rutkin, B. 279

Sakamoto, T. 249, 308 Sano, K. 99, 285 Schaltenbrand, G. 410 Schmidt, K. 24, 329

Schvarcz, J. R. 9, 73 Sekino, H. 99, 285 Shandurina, A. N. 136 Shlbuya, M. 156

Shimabukuro, H. 265 Shimizu, S. 97 Smirnov, V. M. 136 Sramka, M. 193, 244

Sugita, K. 156 
Sugiyama, H. 285 Suzuki, J. 249, 308 Szekely, E. G. 256

Takaoka, Y. 156 Taren, J. A. 165 Teraura, T. 296 Tomlinson, J. D. W. 277 Tóth, S. 49

Tsubokawa, T. 10, 280 Tucker, R. P. 165

Uematsu, S. 79, 187 Umbach, W. 80, 232

Vajda, J. 49 Voris, H. C. 86 
Walker, A. E. 187 Walsh, G. 239 Walter, R. 239 Watanabe, M. 259 Watkins, E. S. 277, 278 Whisler, W. W. 86 Wilson, W. P. 163, 241

Yap, J. 277 Yoshida, T. 291 Yoshii, N. 97 Yoshimasu, N. 99

Zamboni, R. 150 Zarand, P. 49 